Management international

International Management

Gestiòn Internacional

\title{
Le Mentorat envers les Consultants Salariés du Secteur du Conseil en Management : Levier de Fidélisation ou Source d'Opportunités Externes?
}

\section{Mentoring in the Management Consulting Industry: A lever for Loyalty-Building or a Source for External Opportunities? \\ La mentoría en el sector de la consultoría de gestión: ¿un estímulo de la fidelización o una fuente de oportunidades externas?}

\author{
Caroline TILLOU, Ahmed Khalil BEN AYED, Assâad EL AKREMI et Christian \\ VANDENBERGHE
}

\section{Volume 20, numéro 1, automne 2015}

URI : https://id.erudit.org/iderudit/1045356ar

DOI : https://doi.org/10.7202/1045356ar

\section{Aller au sommaire du numéro}

Éditeur(s)

HEC Montréal

Université Paris Dauphine

ISSN

1206-1697 (imprimé)

1918-9222 (numérique)

Découvrir la revue

Citer cet article

TILLOU, C., BEN AYED, A. K., EL AKREMI, A. \& VANDENBERGHE, C. (2015). Le

Mentorat envers les Consultants Salariés du Secteur du Conseil en

Management : Levier de Fidélisation ou Source d'Opportunités Externes ?

Management international / International Management / Gestiòn Internacional, 20(1), 67-77. https://doi.org/10.7202/1045356ar

\section{Résumé de l'article}

Cette étude examine l'impact des pratiques de mentorat auprès des consultants salariés du secteur du conseil en management en France. Les analyses révèlent que l'estime de soi organisationnelle médiatise partiellement une relation positive entre le mentorat et l'engagement affectif envers l'organisation. L'engagement affectif médiatise une relation négative entre le mentorat et l'intention de départ des consultants. Toutefois, ni le mentorat ni l'estime de soi organisationnelle ne sont liés à l'engagement envers la carrière, lequel n'est pas lié à l'intention de quitter l'organisation. Nous discutons la portée de ces résultats pour la compréhension de ces pratiques auprès des travailleurs du savoir.
Tous droits réservés (C) Management international / International Management / Gestión Internacional, 2015
Ce document est protégé par la loi sur le droit d'auteur. L'utilisation des services d’Érudit (y compris la reproduction) est assujettie à sa politique d'utilisation que vous pouvez consulter en ligne. 


\title{
Le Mentorat envers les Consultants Salariés du Secteur du Conseil en Management : Levier de Fidélisation ou Source d'Opportunités Externes?
}

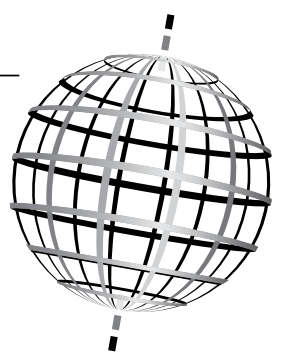

\author{
Mentoring in the Management Consulting Industry: A lever \\ for Loyalty-Building or a Source for External Opportunities?
}

\section{La mentoría en el sector de la consultoría de gestión: $i$ un estímulo de la fidelización o una fuente de oportunidades externas?}

\author{
CAROLINE TILLOU \\ Université de Toulouse \\ Toulouse Business School \\ AHMED KHALIL BENAYED \\ HEC Montréal
}

\author{
ASSÂAD ELAKREMI \\ Université de Toulouse 1 \\ Capitole
}

\author{
CHRISTIAN VANDENBERGHE \\ Département de Managément \\ HEC Montréal
}

\section{RÉSUMÉ}

Cette étude examine l'impact des pratiques de mentorat auprès des consultants salariés du secteur du conseil en management en France. Les analyses révèlent que l'estime de soi organisationnelle médiatise partiellement une relation positive entre le mentorat et l'engagement affectif envers l'organisation. L'engagement affectif médiatise une relation négative entre le mentorat et l'intention de départ des consultants. Toutefois, ni le mentorat ni l'estime de soi organisationnelle ne sont liés à l'engagement envers la carrière, lequel n'est pas lié à l'intention de quitter l'organisation. Nous discutons la portée de ces résultats pour la compréhension de ces pratiques auprès des travailleurs du savoir.

Mots clés : mentorat, engagement, estime de soi organisationnelle, intention de départ, travailleurs du savoir

\section{ABSTRACT}

This study examines the impact of mentoring practices in the management consulting industry in France. Analyses reveal that organizational-based self-esteem partially mediates a positive relationship between mentoring practices and affective commitment while affective commitment mediates a negative relationship between mentoring and consultants' turnover intention. In contrast, mentoring practices and organizational-based self-esteem were unrelated to career commitment, which was also unrelated to turnover intention. We discuss the implications of these results for our understanding of the impact of these practices among knowledge workers.

Keywords: mentoring, commitment, organizational-based self-esteem, turnover intention, knowledge workers

\section{RESUMEN}

Este estudio examina el impacto de las prácticas de mentoría en el sector de la consultoría de gestión en Francia. Los análisis revelan que la autoestima en el ámbito de la organización incide parcialmente en una relación positiva entre las prácticas de mentoría y el compromiso afectivo, mientras que el compromiso afectivo incide en una relación negativa entre las prácticas de mentoría y la intención de abandonar la empresa por parte de los empleados. En cambio, las prácticas de mentoría y la autoestima en el ámbito de la organización no guardan relación alguna con el compromiso profesional, ni este a su vez con la intención de abandonar la empresa. Analizamos qué consecuencias tienen estos resultados en nuestra comprensión del impacto de estas prácticas entre los profesionales del conocimiento.

Palabras Claves: mentoría, compromiso, autoestima en el ámbito de la organización, intención de abandonar la empresa, profesionales del conocimiento
Cituation de crise ou de reprise économique, le départ volontaire des salariés est très souvent une préoccupation réelle des dirigeants d'entreprise. Selon le cabinet d'audit et de conseil Deloitte, «pour prévenir la perte des talents généralement constatée en période de reprise économique caractérisée par un "tsunami de candidatures", les managers doivent éviter les erreurs qui peuvent développer l'intention de départ des salariés» (Chaftez, Adair Erickson et Ensell, 2011). La fidélisation des salariés est bien envisagée comme une politique stratégique pour le développement et la survie des entreprises. La mesure du taux de roulement représente ainsi un indicateur clé de la gestion des talents (Bersin \& Associates, 2009). Ce constat est d'autant plus vrai pour les entreprises, tels les cabinets conseil, employant des travailleurs du savoir, en l'occurrence ici des consultants salariés. En effet, ces travailleurs du savoir développent et partagent des connaissances et un savoir-faire nécessaires à la création d'un avantage concurrentiel : ces salariés constituent un socle fondamental au succès organisationnel (Anand, Gardner et Morris, 2007). C'est la raison pour laquelle les cabinets de conseil, forts du capital humain qu'ils 
détiennent, sont devenus un véritable terrain de recherche en sciences de gestion (Sarvary, 1999; Werr et Stjernberg, 2003).

De nombreux travaux se sont interrogés sur le rôle des pratiques de gestion de ressources humaines dans le processus de fidélisation (Allen, Shore et Griffeth, 2003; Batt, 2002; Kehoe et Wright, 2013; Sun, Aryee et Law, 2007). Toutefois, peu d'entre eux sont consacrés au cas des travailleurs du savoir (Tillou et Igalens, 2012). Cette recherche s'inscrit dans cette perspective en développant un modèle centré sur les effets d'une pratique particulièrement adaptée à ce type de travailleurs : le mentorat. Cette pratique, reflétant un soutien de l'employé dans son processus d'apprentissage lui permettant de développer son potentiel (Ragins et McFarlin, 1990), est à l'origine de la relation mentor-protégé et est essentielle au partage du savoir dans les cabinets de conseil (Swart et Kinnie, 2003). Nous proposons donc d'analyser le processus de départ volontaire des consultants des cabinets de conseil grâce à l'étude des effets de la relation mentor interne-protégé.

Notre étude vise deux objectifs importants. Tout d'abord, alors que les travaux antérieurs ont mis en évidence le rôle du mentorat dans le développement de l'engagement organisationnel affectif et la rétention des employés (p.ex. Aryee et Chay, 1994; Hartmann, Rutherford, Hamwi, et Friend, 2013; Payne et Huffman, 2005; Richard, Ismail, Bhuian, et Taylor, 2009), nous élargissons cette perspective en considérant l'engagement envers la carrière comme un autre médiateur potentiel. En effet, le mentorat favorise l'adoption des valeurs organisationnelles (Viator et Scandura, 1991), ce qui se traduit par un engagement affectif plus marqué. Cependant, comme le mentorat vise le développement des compétences, il peut ouvrir de nouvelles perspectives d'évolution et de carrière (Haggard, Dougherty, Turban, et Wilbanks, 2011; Scandura, 1992), renforçant ainsi l'engagement envers la carrière, et mener à une mobilité accrue sur le marché. Cet effet peut être particulièrement présent parmi les travailleurs du savoir, lesquels sont reconnus pour leur mobilité professionnelle (Alvesson, 2000). Notre étude considère donc l'engagement organisationnel affectif et l'engagement envers la carrière comme deux médiateurs alternatifs du mentorat.

Notre deuxième objectif consiste à étudier l'estime de soi organisationnelle (Pierce et Gardner, 2004) comme un médiateur venant s'insérer entre le mentorat et l'engagement organisationnel affectif et envers la carrière. L'estime de soi organisationnelle reflète la valeur que les individus s'accordent en termes de compétences et d'importance personnelle au sein de leur organisation (Pierce, Gardner, Cummings, et Dunham, 1989). En nous centrant sur les travailleurs du savoir, nous nous intéressons à des salariés le plus souvent conscients de leurs atouts et exigeants envers leur employeur (Alvesson, 2000). Par conséquent, leur niveau d'estime personnelle constitue une source d'influence potentielle sur l'intention de départ. Nous pensons donc que le mentorat exercera ses effets sur l'engagement affectif et envers la carrière (et finalement sur l'intention de départ) partiellement au travers d'une estime de soi plus élevée. Cette étude répond à des questions soulevées quant au rôle de l'estime de soi au travail (Bowling, Eschleman, Wang, Kirkendal et Alarcon, 2010) et est la première à tester de tels liens dans l'analyse du comportement de départ volontaire des travailleurs du savoir.

\section{Modèle Théorique et Hypothèses}

Par définition, le mentorat représente un outil de développement de carrière qui répond à des besoins de renforcement des compétences (Aryee et Chay, 1994; Haggard et al., 2011; Hunt et Michael, 1983). À ce titre, le mentorat est une pratique particulièrement adaptée aux consultants. En effet, en tant que travailleurs du savoir, les consultants y voient une opportunité non seulement d'assurer une qualité de service auprès de leurs clients mais aussi d'ouvrir des perspectives de carrière. De nombreux cabinets de conseil offrent d'ailleurs le soutien d'un mentor interne dès le recrutement des consultants. Ces expériences présentent des résultats positifs tant pour le mentor que pour le protégé et son organisation (Udeh et Omar, 2009).

Le mentor est un individu qui utilise son expérience pour assister son protégé au sein de l'organisation (Haggard et al., 2011; Kram, 1985) et assurer son développement personnel et professionnel (Haggard et al., 2011; Ragins et Cotton, 1999). Celui-ci remplit deux fonctions principales : une fonction psychosociale et une autre, davantage liée à la carrière de son protégé (Kram, 1985). D’une part, la fonction psychosociale lui permet d'apporter des conseils au protégé, de l'aider à définir son rôle, de l'encourager et de créer des relations plus informelles avec lui. Ces interventions renforcent des facteurs personnels tels que l'amour-propre du protégé mais aussi des facteurs directement liés au travail tels que ses compétences, son efficacité et son identité dans l'organisation. D’autre part, en s'impliquant dans l'évolution de la carrière du salarié, le mentor lui offre son expertise mais aussi une visibilité auprès de son réseau de contacts ou encore des missions ambitieuses (Kram, 1985). Le protégé peut ainsi profiter de l'expérience et de la position hiérarchique de son mentor.

\section{Mentorat, ENGAgEMENT AFFECTIF, ET INTENTION DE DÉPART}

La plupart des études sur le mentorat ont été consacrées à l'analyse des effets du mentorat sur les attitudes et les comportements du protégé ainsi que sur son évolution de carrière (Allen, Eby, Poteet, Lentz, et Lima, 2004; Allen, Eby, O'Brien, et Lentz, 2008). Par exemple, le soutien du mentor facilite la mobilité des protégés (Day et Allen, 2004; Fagenson, 1989) et l'atteinte de leurs objectifs de carrière. La réussite des protégés est d'autant plus forte qu'ils sont suivis par leur mentor (Eby et al., 2008; Joiner, Bartram, et Garreffa, 2004). Certaines études ont aussi montré l'impact $\mathrm{du}$ mentorat sur l'engagement organisationnel et le taux de roulement des salariés (Allen et al., 2004; Fleig-Palmer, 2009; Payne et Huffman, 2005; Udeh et Omar, 2009; Viator et Scandura, 1991). La plupart des auteurs se sont attachés à comparer les niveaux d'engagement des salariés qui ont un mentor à ceux qui n'en ont pas, à mesurer l'impact de la qualité de la relation sur l'engagement organisationnel du pro- 
tégé, à identifier les fonctions les plus influentes du mentorat, ou encore à évaluer l'influence des conditions de la relation (supérieur hiérarchique ou pas) (Allen et al., 2004; Aryee et Chay, 1994; Dawley, Andrews, et Bucklew, 2008; Payne et Huffman, 2005).

L'engagement organisationnel affectif est probablement plus en lien avec le mentorat que d'autres formes d'engagement car les pratiques de mentorat amènent les protégés à se socialiser vis-à-vis des valeurs de l'organisation (Viator et Scandura, 1991). Or, l'engagement affectif repose sur une identification par rapport aux valeurs de l'organisation (Meyer, Becker, et Vandenberghe, 2004). D’une part, selon les principes d'échange social, dans la mesure où le mentorat est perçu comme une pratique positive par le protégé, ce dernier fait preuve de réciprocité en témoignant d'un engagement envers l'organisation qui lui a offert cette opportunité de développement. Le mentorat soulage aussi en partie le protégé de son souci de gestion de carrière. Enfin, la qualité de la relation avec le mentor encourage le protégé à devenir «le reflet de son modèle» en affichant ce type d'attitudes positives envers l'organisation (Payne et Huffman, 2005). D'autre part, le mentorat fait partie des pratiques de socialisation (Dawley et al., 2008). Le protégé profite donc de la proximité relationnelle avec le mentor pour adopter les valeurs de l'entreprise (Viator et Scandura, 1991).

Par ailleurs, comme les travaux empiriques ont confirmé l'existence d'une relation négative entre l'engagement affectif et l'intention de partir (Tett et Meyer, 1993; Vandenberghe, Bentein, et Stinglhmaber, 2004), on peut présumer que le mentorat agira indirectement (négativement) sur l'intention de partir par l'intermédiaire de l'engagement affectif. Ce processus prend une dimension stratégique dans le cas des travailleurs du savoir. Selon Kinnear et Sutherland (2000), l'engagement organisationnel de ces salariés est essentiel car ils sont très mobiles sur le marché du travail. Ces développements théoriques nous amènent à formuler les hypothèses suivantes :

Hypothèse $1 a$ : Les pratiques de mentorat sont positivement liées à l'engagement organisationnel affectif des consultants.

Hypothèse $1 b$ : L'engagement organisationnel affectif des consultants médiatise une relation négative entre les pratiques de mentorat et l'intention de départ.

\section{MENTORAT, ENGAGEMENT ENVERS LA CARRIÈRE, ET INTENTION DE DÉPART}

Les travailleurs intellectuels sont souvent présentés comme des experts autonomes, réticents à la hiérarchie et volatiles. Davantage impliqués à l'égard de leur travail que de leur organisation, leur profil atypique suscite des interrogations managériales (Horwitz, Heng, et Quazi, 2003). Un tel constat suggère que les travailleurs du savoir pourraient utiliser le levier du mentorat pour développer un engagement envers leur carrière, et ainsi chercher à quitter leur employeur afin de rentabiliser leurs compétences ailleurs. L'engagement envers la carrière se distingue de l'implication au travail ou de l'engagement à l'égard de l'organisation (Blau, 1985; Hall, 1971). Meyer, Allen, et Smith (1993) établissent ainsi une distinction entre l'implication envers un métier et l'ambition d'accéder à un poste supérieur (cf. Ellemers, De Gilder, et van den Heuvel, 1998). L'engagement envers la carrière reflète ainsi le désir de poursuivre des objectifs de carrière et incite les salariés à s'investir dans leur travail pour atteindre un niveau de performance essentiel à leur avancement personnel (Den Hartog et Belschak, 2007).

D'un point de vue conceptuel, plusieurs processus contribuent à faire du mentorat un antécédent de l'engagement envers la carrière (Allen et al., 2004; Haggard et al., 2011). Tout d'abord, le mentor s'emploie dans le développement de la carrière et l'accompagnement psychosocial de son protégé. La première fonction renforce les attitudes à l'égard de la carrière telles que l'engagement et l'autoréalisation des protégés, alors que la seconde enrichit les réseaux et par conséquent, les opportunités à l'extérieur de l'organisation. Aussi, les protégés trouvent chez leur mentor une expérience qui les inspire, un modèle. L'apprentissage social renforce les fondements de cette relation personnelle (Kram, 1986) Le protégé peut s'appuyer sur cette proximité pour faire part de ses besoins et réaliser ses ambitions de carrière. De plus, même si le développement de l'engagement envers la carrière peut requérir davantage de temps que celui d'autres formes d'engagement, l'insécurité professionnelle propre à la conjoncture actuelle lui ont donné toute son importance. En effet, l'engagement envers la carrière apporte la continuité pouvant faire défaut à l'équilibre professionnel des salariés (Aryee et Chay, 1994; Chang, 1999). Bien qu'il soit difficile de dissocier les motivations liées au travail et à la carrière (Romzek, 1989), cette continuité se traduit en restant au sein de l'organisation ou en la quittant pour poursuivre ses objectifs professionnels dans un autre environnement.

D'un point de vue empirique, Blau (1985) rapporte une relation non significative entre l'engagement envers la carrière et l'intention de départ mais dans une étude subséquente (Blau, 1989) il trouve une relation positive entre les deux construits. Ce dernier résultat a été confirmé par d'autres études (Aryee et Tan, 1992; Bedeian, Kemery, et Pizzolatto, 1991; Chang, 1999). Néanmoins, certaines conditions peuvent nuancer ces conclusions. Dans le cas où les opportunités de développement au sein de l'organisation sont faibles, les salariés à fort engagement de carrière pourraient envisager de quitter leur employeur (Bedeian et al., 1991). Les spécificités de la population des consultants telles que leur mobilité et leur intérêt pour le succès de carrière peuvent constituer des circonstances similaires et ceci d'autant plus que le soutien du mentor aura fait émerger de nouvelles perspectives de carrière pour les protégés. Ceci nous amène à envisager l'engagement envers la carrière des consultants comme une source d'incitation au départ volontaire. Nous proposons donc les hypothèses suivantes :

Hypothèse $2 a$ : Les pratiques de mentorat sont positivement liées à l'engagement envers la carrière des consultants. 
Hypothèse $2 b$ : L'engagement envers la carrière des consultants médiatise une relation positive entre les pratiques de mentorat et l'intention de départ.

\section{LE RÔLE MÉDIATEUR DE L'ESTIME DE SOI ORGANISATIONNELLE}

Nous postulons que la relation entre mentorat et engagement affectif et envers la carrière sera partiellement médiatisée par l'estime de soi organisationnelle. De par les fonctions qui lui sont reconnues, le mentor développe une relation qui renforce l'estime de soi de son protégé (Kram, 1983; Koberg, Boss, et Goodman, 1998). L'estime de soi organisationnelle représente «l'évaluation de l'employé quant à son adéquation personnelle et sa valeur en tant que membre de l'organisation » (Gardner et Pierce, 1998, p. 50). Elle se construit progressivement à partir des expériences personnelles vécues au sein de l'organisation et des échanges avec d'autres membres tels que les collègues et le supérieur (Pierce, Gardner, Dunham, et Cummings, 1993).

Le caractère médiateur de l'estime de soi s'explique de deux manières. Tout d'abord, la relation de mentorat nourrit l'estime des consultants : plus les salariés sont guidés par leur mentor, plus leur estime de soi organisationnelle sera renforcée. Aussi, l'estime de soi organisationnelle reflète une satisfaction personnelle provenant de la relation avec l'entourage professionnel, ce qui amènerait le consultant à développer son engagement organisationnel (Lee et Peccei, 2007; Meyer et Allen, 1997) mais aussi envers sa carrière (Carson, Carson, Lanford, et Roe, 1997). D'une part, toute forme de soutien de l'organisation (ou de ses représentants), tel le mentorat, augmente le sentiment d'appartenance (Ferris, Brown, et Heller, 2009) et l'engagement affectif (Lee et Peccei, 2007). D'autre part, le mentorat augmente la confiance de l'individu en ses compétences et renforce le sentiment d'appartenance à la profession de consultant et donc l'engagement de carrière. La satisfaction issue du mentorat encouragerait le consultant dans ses perspectives professionnelles et renforcerait son engagement de carrière.

Hypothèse $3 a$ : Les pratiques de mentorat sont positivement liées à l'estime de soi organisationnelle des consultants.

Hypothèse $3 b$ : L'estime de soi organisationnelle des consultants médiatise une relation positive entre les pratiques de mentorat et l'engagement organisationnel affectif.
Hypothèse $3 c$ : L'estime de soi organisationnelle des consultants médiatise une relation positive entre les pratiques de mentorat et l'engagement de carrière.

La Figure 1 reprend l'ensemble des hypothèses de l'étude.

\section{Méthode}

\section{ECHANTILLON ET PROCÉDURE}

Les données de cette étude sont issues d'une recherche plus large sur les pratiques de gestion, l'engagement, et l'intention de départ dans les cabinets de consultants en France. Cette enquête fut réalisée en deux temps. Les deux questionnaires furent envoyés par courrier électronique par le SYNTEC Conseil en Management (i.e., un syndicat professionnel représentant $60 \%$ du marché des activités de conseil en stratégie et management) aux dirigeants des cabinets de conseil qui, à leur tour ont relayé notre requête à leurs salariés. Ceux-ci accédaient directement au site de l'enquête. Le premier questionnaire mesurait la perception des pratiques de mentorat et l'estime de soi organisationnelle. Quatre mois plus tard, un deuxième questionnaire a permis de mesurer l'engagement organisationnel et de carrière et l'intention de départ. Au temps 1, nous avons recueilli 250 réponses, parmi lesquelles 196 consultants (78.4\%) bénéficiant d'un programme de mentorat au sein de leur cabinet. Seules ces personnes ont été retenues pour cette étude. Parmi elles, 188 ont répondu au temps 2 . Les répondants provenaient de 15 cabinets $(M=12.53$ par cabinet; $\min .=3$, $\max .=56)$ et comprenaient $41.7 \%$ de femmes. L'âge moyen était de 32.89 ans $(E T=7.96)$ et l'ancienneté de $3.81(E T=4.00)$.

\section{Mesures}

Pratiques de mentorat. Nous avons mesuré la perception des pratiques de mentorat avec l'échelle de Ragins et McFarlin (1990), lesquels se sont inspirés de Kram (1985) pour développer un instrument dénommé Mentor Role Instrument (MRI). Sur base de nos échanges avec les représentants $\mathrm{du}$ SYNTEC, nous avons retenu 5 des 11 dimensions de l'échelle en raison de leur pertinence pour les consultants. Ces dimensions concernent l'intervention du mentor en tant que modèle (p.ex. "Mon mentor représente bien celui que je souhaite devenir»), protecteur (p.ex. "Mon mentor intervient pour moi en cas de problème au sein du cabinet»),

\section{FIGURE 1 Modèle de recherche et hypothèses}

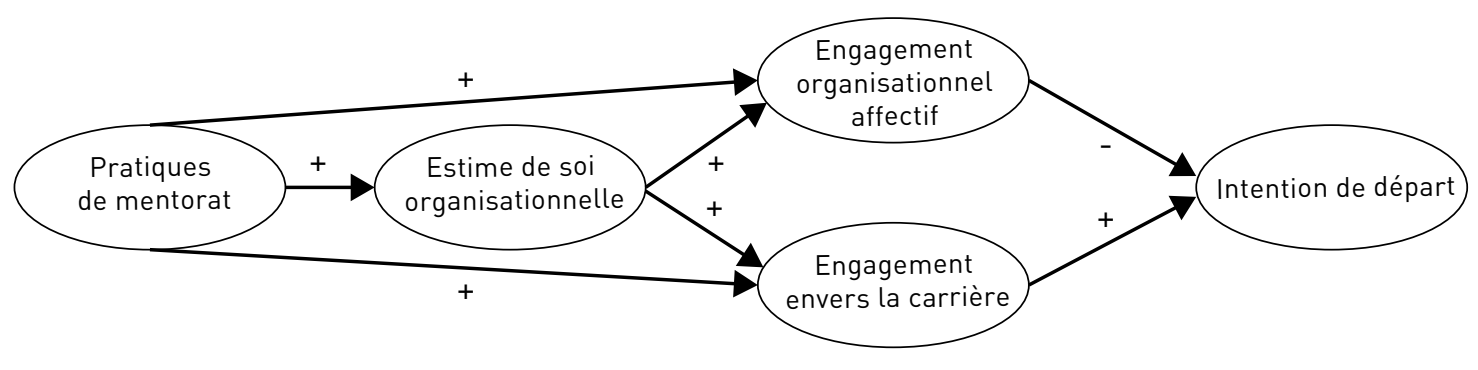


coach (p.ex. «Mon mentor me propose des stratégies claires pour atteindre mes objectifs de carrière»), ami (p.ex. «Mon mentor est quelqu'un à qui je peux me confier»), et conseiller (p.ex. «Mon mentor guide mon évolution professionnelle»). Chaque dimension contient 3 items. Les 5 dimensions renvoient à 2 domaines : la perception quant (a) à l'aspect informel de la relation avec le mentor (rôle d'ami et protecteur) et (b) au rôle du mentor dans le développement personnel et professionnel (rôle de coach, modèle, et conseiller). Ces deux aspects sont en effet présents dans le discours des dirigeants : le premier est mis en avant pour faciliter l'intégration des consultants et favoriser leur confort lors du retour de mission dans l'organisation; le second rappelle le besoin de formation et d'apprentissage attendu par le consultant tout au long de son parcours. Enfin, certains auteurs ont utilisé des versions courtes du MRI (Wanberg, Welsh, et KammeyerMueller, 2007). Cette échelle a une fiabilité élevée dans notre étude $(\mathrm{a}=.94)$.

Estime de soi organisationnelle. L'échelle retenue comprenait initialement 10 items (Pierce et al., 1989) mais, soucieux de la longueur du questionnaire, nous avons opté pour une version à 6 items, validée par Chattopadhyay (1999) (a $=.81$ ) (p. ex. : «Je suis quelqu'un qui compte pour les personnes qui travaillent dans ce cabinet»).

Engagement organisationnel affectif et de carrière. L'engagement organisationnel a été mesuré par la version française (Vandenberghe et al., 2004) de l'échelle de Meyer et al. (1993) (6 items; a =.88), alors que l'engagement de carrière a été mesuré par l'échelle de Ellemers et al. (1998; p.ex. : «Les principales ambitions de ma vie sont liées à ma carrière»; $\mathrm{a}=.87$ ).

Intention de départ. L'intention de quitter a été mesurée par la version française (Vandenberghe et al., 2004) de l'échelle en 3 items de Jaros (1997) (p.ex. : «J'ai l'intention de chercher un emploi dans un autre cabinet de conseil dans l'année qui vient»; $\mathrm{a}=.85$ ).

\section{Résultats}

\section{ANALYSES FACTORIELLES CONFIRMATOIRES}

Nous avons procédé à l'analyse factorielle confirmatoire du modèle de mesure (mentorat, estime de soi, engagement organisationnel et de carrière, et intention de départ) avec le logiciel Mplus. Afin de simplifier notre modèle, lequel contenait 35 items, nous avons créé 4 indicateurs pour la variable de mentorat. Ces indicateurs ont été établis en combinant 2 ou 3 items de l'échelle de mentorat de manière aléatoire (cf. Little, Cunningham, Shahar, et Widaman, 2002). Comme l'indique le tableau 1, l'analyse confirme la très bonne adéquation du modèle aux données $\left(c^{2}=343.51 ; p<.01\right.$; CFI =.92; RMSEA =.06). Par ailleurs, nous avons comparé ce modèle à des modèles alternatifs en combinant 2 ou plusieurs facteurs en un seul. Suivant le test de différence de khi-carré (Bentler et Bonett, 1980), les résultats (tableau 1) indiquent qu'aucun modèle alternatif ne présente une valeur significativement meilleure que celle du modèle théorique. Ceci indique que notre modèle théorique représente la meilleure solution factorielle.

\section{STATISTIQUES DESCRIPTIVES ET CORRÉLATIONS}

Le mentorat corrèle positivement avec l'engagement affectif $(r=.32, p<.01)$ et l'estime de soi $(r=.28, p<.01)$, et l'engagement affectif corrèle positivement avec l'engagement de carrière $(r=.15, p<.05)$ et l'estime de soi $(r=.32, p<.01)$. Enfin, le mentorat, l'estime de soi, et l'engagement affectif corrèlent négativement avec l'intention de départ $(r=-.30, p<.01, r=$ $-.18, p<.05$, et $r=-.58, p<.01$ ) (tableau 2 ).

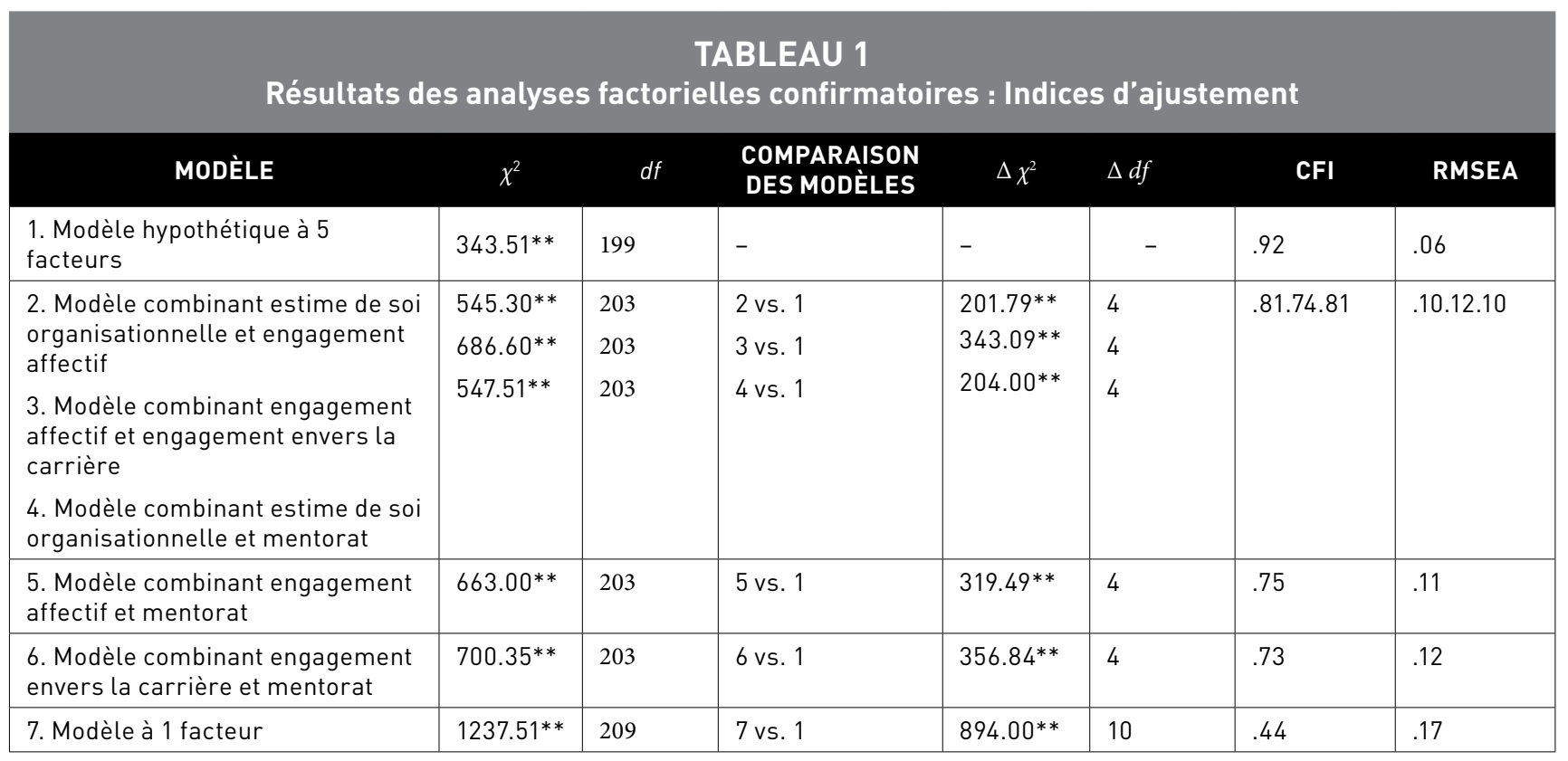

Note. $\mathrm{N}=178$. $\mathrm{CFI}=$ comparative fit index; RMSEA = root-mean-square error of approximation. $* * p<.01$. 


\begin{tabular}{|c|c|c|c|c|c|c|c|}
\hline & Stat & te de & $\begin{array}{l}\text { AU } 2 \\
\text { res et c }\end{array}$ & lations & & & \\
\hline VARIABLE & $M$ & ET & 1 & 2 & 3 & 4 & 5 \\
\hline 1. Pratiques de mentorat & 3.20 & 0.64 & $(.94)$ & 0.10 & $0.28 * *$ & $0.32 * *$ & $-0.30 * *$ \\
\hline 2. Engagement envers la carrière & 3.08 & 0.71 & 0.15 & (.81) & 0.11 & $0.15^{*}$ & 0.03 \\
\hline 3. Estime de soi organisationnelle & 3.34 & 0.46 & $0.36^{* *}$ & 0.11 & (.89) & $0.32 * *$ & $-0.18^{*}$ \\
\hline 4. Engagement affectif & 3.30 & 0.70 & $0.23^{* *}$ & $0.37^{* *}$ & $0.35^{* *}$ & (.87) & $-0.58 * *$ \\
\hline 5. Intention de départ & 2.63 & 1.37 & $-0.31 * *$ & -0.01 & -0.17 & $-0.67 * *$ & $(.84)$ \\
\hline
\end{tabular}

Note. $N=188$. Les corrélations bivariées sont rapportées au-dessus de la diagonale alors que les corrélations obtenues pour l'analyse factorielle confirmatoire par le biais de Mplus sont rapportées en-dessous de la diagonale. Les coefficients alpha de Cronbach sont rapportés entre parenthèses sur la diagonale du tableau.

${ }^{*} p<.05 ;{ }^{* *} p<.01$.

\section{TEST DU MODÈLE STRUCTUREL}

Ensuite, nous avons procédé au test du modèle structurel spécifié dans la figure 1 . Nous avons créé un seul indicateur par construit, et les saturations des indicateurs ont été fixées à la racine carrée du coefficient alpha de Cronbach pour l'échelle correspondante, multipliée par la variance de l'échelle. L'ajustement du modèle est excellent $\left(\chi^{2}[2]=4.58\right.$, $n s, \mathrm{CFI}=.98$; RMSEA =.09). Nous avons comparé ce modèle à un modèle alternatif dans lequel un lien a été ajouté entre l'estime de soi et l'intention de départ mais ce modèle n'améliorait pas le modèle théorique $\left(\Delta \chi^{2}[1]=2.88, n s\right)$. De même, un modèle ajoutant un lien entre le mentorat et l'intention de départ n'améliorait pas le modèle théorique non plus $\left(\Delta \chi^{2}\right.$ $[1]=0.62, n s)$. Donc, notre modèle structurel théorique est retenu comme le modèle final pour le test des hypothèses.

\section{TEST DES HYPOTHÈSES}

Les paramètres du modèle structurel sont présentés dans la figure 2. Le mentorat est positivement lié à l'engagement organisationnel $(b=.24, p<.01)$, confirmant l'hypothèse la. D'autre part, comme l'engagement organisationnel est négativement lié à l'intention de départ $(b=-.63, p<.01)$, il est vraisemblable qu'il médiatise la relation entre le mentorat et l'intention de départ. Pour examiner cette possibilité, nous avons utilisé une procédure de bootstrap permettant de calculer un intervalle de confiance pour la valeur de l'effet indirect du mentorat sur l'intention de départ via l'engagement affectif à partir de 1000 rééchantillonnages (avec remise) de l'échantillon original (MacKinnon, Lockwood, et Williams, 2004). Comme l'intervalle de confiance obtenu ne contient pas zéro (effet indirect $=-.15$, intervalle de confiance à $95 \%$ $=-.37,-.06)$, l'effet indirect est significatif. L'hypothèse $1 \mathrm{~b}$ est confirmée. Par contre, il n'y a pas de relation entre le mentorat et l'engagement de carrière $(b=.10, n s)$ et entre l'engagement de carrière et l'intention de départ $(\mathrm{b}=.12, n s)$. Dès lors, les hypothèses $2 \mathrm{a}$ et $2 \mathrm{~b}$ sont rejetées.

La figure 2 montre aussi que le mentorat est positivement lié à l'estime de soi $(\mathrm{b}=.35, p<.01)$, confirmant l'hypothèse 3a. D'autre part, comme l'estime de soi est positivement liée à l'engagement affectif $(b=.32, p<.01)$, il est vraisemblable qu'elle médiatise la relation entre le mentorat et l'engagement affectif. Suivant la même procédure de bootstrap que celle décrite ci-dessus, nous pouvons établir que cette médiation est significative (effet indirect $=.11$, intervalle de confiance à $95 \%=.05, .19)$. L'hypothèse $3 \mathrm{~b}$ est confirmée. En revanche, comme l'estime de soi n'est pas liée à l'engagement de carrière $(\mathrm{b}=.06, n s)$, l'hypothèse $3 \mathrm{c}$ qui spécifiait une médiation de l'estime de soi dans la relation entre mentorat et engagement de carrière est rejetée. Enfin, des analyses complémentaires révèlent un effet indirect significatif de l'estime de soi sur l'intention de départ via l'engagement organisationnel (effet indirect $=-.20$, intervalle de confiance à $95 \%=-.62$, -.15). Nous avons aussi testé la double médiation des effets du mentorat sur l'intention de départ via l'estime de soi et l'engagement affectif. Cette double médiation est significative (effet indirect $=-.07$, intervalle de confiance à $95 \%=$ $-.18,-.04)$.

\section{FIGURE 2}

Paramètres structuraux complètement standardisés associés au modèle théorique

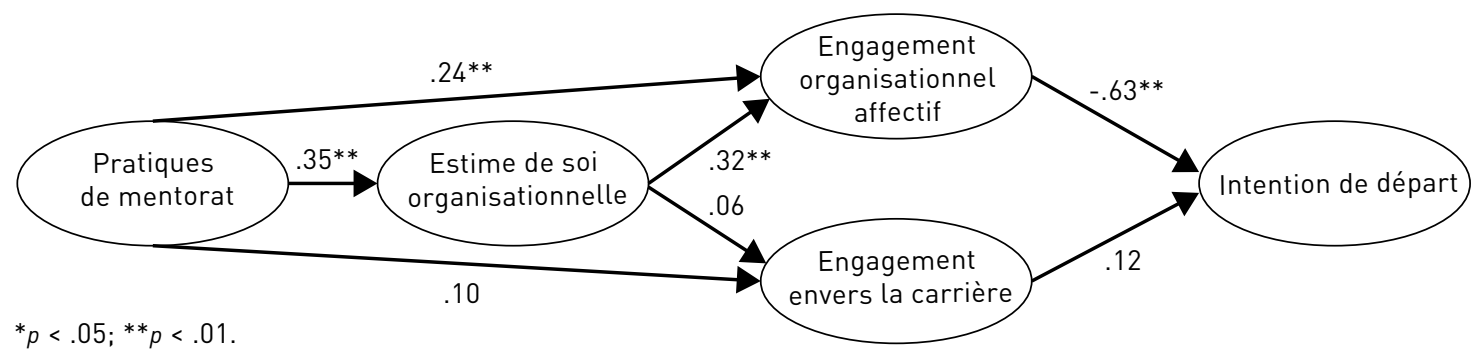




\section{Discussion}

Cette étude, réalisée dans une perspective de psychologie organisationnelle et utilisant une méthodologie quantitative, avait pour objectif d'examiner l'impact des pratiques de mentorat au sein des cabinets de conseil en management. Ce faisant, cette étude ne se penche pas sur les stratégies d'acteurs poursuivies par les mentors et les consultants mais montre que sur le plan individuel la relation mentor-protégé permet de limiter l'intention de départ des consultants via ses effets sur l'estime de soi organisationnelle et l'engagement organisationnel affectif. De plus, les résultats montrent que l'engagement de carrière n'a pas d'effet significatif.

\section{ENGAGEMENT ORGANISATIONNEL AFFECTIF VS ENGAGEMENT ENVERS LA CARRIÈRE}

Nos conclusions apportent une validation partielle au cadre conceptuel établi dans cette recherche. Celui-ci reposait sur l'idée selon laquelle le mentorat développe l'engagement organisationnel affectif et l'engagement de carrière des consultants, lesquels agiraient de manière opposée sur l'intention de départ. Premièrement, les effets directs liés à l'engagement organisationnel sont significatifs. La validation de ces hypothèses fait écho au rôle majeur de l'échange social dans le contexte de la fidélisation des salariés. Si de tels résultats ont déjà été constatés empiriquement (Payne et Huffman, 2005), cette étude étend l'effet de réciprocité au cas des consultants. En effet, les consultants sont sensibles à la pratique du mentorat dans la mesure où ils répondent par un attachement émotionnel plus fort à l'égard de leur cabinet de conseil, ce qui limite leur intention de départ. Au-delà de cet effet de réciprocité, le mentorat représente une pratique particulière. Tout en jouant son rôle dans le développement et l'accompagnement du consultant, le mentor facilite l'attachement à l'organisation. Le mentor, comme le supérieur hiérarchique, peut refléter les valeurs, l'expérience, et les attentes de l'organisation. En d'autres termes, en personnifiant le cabinet de conseil, la pratique de mentorat dépasse le cadre de la relation individuelle pour créer un lien affectif avec l'organisation.

Deuxièmement, les résultats de cette étude rejettent l'idée selon laquelle les pratiques de mentorat développent l'engagement des consultants envers leur carrière, lequel augmenterait leur intention de départ. Aucune de ces relations n'est significative. Conformément à la définition de l'engagement envers la carrière dans cette étude (Den Hartog et Belschak, 2007), la relation mentor-protégé n'inciterait pas les consultants à persister dans la réussite de leurs projets de carrière (mais plutôt à renforcer le lien avec l'organisation). Ce constat peut surprendre dans la mesure où la relation créée entre le mentor et le protégé dépasse le cadre strictement lié à la fonction actuelle du protégé et vise davantage son intégration et son développement personnel et professionnel. Une explication serait que l'engagement du salarié varie selon la source du soutien perçu (Bishop, Scott, Goldsby, et Cropanzano, 2005). Dans le cas du mentorat, la source du soutien peut être soit le mentor lui-même soit l'organisation à l'origine de l'usage de cette pratique. La carrière repré- sente une cible d'engagement plus éloignée de la source de soutien (ici, l'organisation ou le mentor), ce qui expliquerait l'absence de rôle du mentorat par rapport à l'engagement de carrière. Par ailleurs, selon Ragins, Cotton, et Miller (2000), la durée de la relation mentor-protégé conditionne l'offre de perspectives de carrière. Or, nous pouvons nous demander si le métier de consultant permet le développement de ces relations durables. Les résultats laissent plutôt entrevoir le mentorat comme étant une source d'attachement recherchée par ces professionnels lors de leur retour de mission dans le cabinet de conseil. Cependant, nous ne détenons pas d'information relativement à la durée de la relation mentor-protégé vécue par les consultants de notre échantillon, ce qui nous empêche de confirmer cette interprétation.

Il serait donc utile à l'avenir de mesurer aussi bien la durée de cette relation dyadique que certains construits tels que le soutien perçu de la part du mentor et de l'organisation. Aussi, la faiblesse de ces résultats rappelle le besoin d'étudier les effets du mentorat sur l'engagement de carrière en dissociant l'impact des différents rôles attribués au mentor (Baranik, Roling, et Eby, 2010; Payne et Huffman, 2005). En effet, que ce soit en distinguant le rôle formel du rôle informel, celui de guide dans le développement de la carrière de celui d'accompagnateur psychosocial, ou simplement en mesurant l'effet des 11 dimensions de l'échelle de Ragins et McFarlin (1990), une approche plus affinée du construit devrait permettre d'identifier certains rôles pouvant développer l'implication des consultants envers leur projet de carrière.

Les effets de l'engagement envers la carrière sur l'intention de départ ne sont pas significatifs : cette variable n'exerce pas de rôle médiateur dans la relation indirecte entre le mentorat et l'intention de départ. D'autres études font état de résultats tout aussi surprenants (p.ex. Ghosh et Reio, 2013). Pour interpréter plus précisément ces résultats, nous pouvons transposer les considérations de Bishop, Scott, et Burroughs (2000) à notre étude. Ainsi, l'engagement des consultants influencerait leur intention de départ dès lors que la cible de l'engagement est proche et concernée par l'intention de départ et que l'intention de départ a un effet immédiat sur l'objet de l'engagement. Or, la carrière ne représente pas une cible d'engagement dont les effets sont suffisamment "immédiats" sur l'intention de départ des consultants et de plus, l'intention de départ n'a pas un effet immédiat sur leur carrière. Enfin, une autre voie d'interprétation consiste à considérer la diversité des répondants de notre échantillon. Tous les consultants interrogés n'occupent pas des postes leur offrant des perspectives de carrière. A ce sujet, Jayasingam et Yong (2013) ont constaté que les effets des pratiques de gestion varient suivant le niveau du poste occupé. Ainsi, les caractéristiques propres à notre échantillon (poste occupé) pourraient avoir un impact sur le lien entre mentorat et engagement de carrière.

\section{L'ESTIME DE SOI ORGANISATIONNELLE DES CONSULTANTS}

Le second objectif de cette recherche consistait à mesurer l'effet médiateur de l'estime de soi organisationnelle des consultants dans la relation entre les pratiques de mentorat 
et l'engagement organisationnel affectif d'une part, et l'engagement de carrière d'autre part. Les résultats ont confirmé ce rôle dans la première relation et l'ont rejeté dans la seconde. Par conséquent, le mentorat développe l'attachement affectif des consultants envers leur cabinet de conseil de par la satisfaction personnelle qu'ils retirent de leur relation avec leur mentor et le soutien organisationnel qui s'exprime dans cette relation. Ainsi, grâce aux connaissances et aux compétences acquises en côtoyant leur mentor, cette relation privilégiée développe leur sentiment d'importance au sein de leur entreprise. Des analyses complémentaires révèlent aussi une double médiation, soit des effets du mentorat sur l'intention de départ via l'estime de soi organisationnelle et l'engagement organisationnel affectif. Si ces relations ont déjà été confirmées indépendamment les unes des autres (Bentein, Stinglhamber, et Vandenberghe, 2000; Bowling et al., 2010; Meyer, Stanley, Herscovitch, et Topolnytsky, 2002; Pierce et Gardner, 2004), cette double médiation offre une compréhension approfondie de la contribution des pratiques de mentorat dans le processus de fidélisation des consultants.

Enfin, selon les résultats de cette étude, l'estime de soi organisationnelle n'influence pas de manière significative l'engagement de carrière des consultants. En d'autres termes, la valeur que le consultant s'attribue en tant que membre de l'organisation ne conditionne pas son engagement de carrière. Ces résultats vont à l'encontre de plusieurs travaux antérieurs (Carson et al., 1997; Pierce et Gardner, 2004; Ragins et al., 2000). Une explication plausible pour ce résultat serait que les consultants de notre échantillon sont des travailleurs salariés et, en raison de leur contrat de travail, bénéficieraient de divers avantages matériels offerts par leur organisation, ce qui indirectement renforcerait le lien avec l'organisation plutôt qu'avec la carrière comme telle. Les résultats montrent en effet que la valeur que le consultant s'attribue en tant que membre du cabinet de conseil (laquelle peut être conditionnée par les avantages matériels offerts par l'organisation) joue un rôle majeur dans son attitude envers le cabinet (engagement organisationnel). Nos résultats auraient aussi pu être différents si le mentor provenait d'un réseau de professionnels extérieur aux cabinets conseil. Il serait par ailleurs intéressant de tester ces relations auprès d'autres professionnels du savoir.

\section{IMPLICATIONS MANAGÉRIALES}

Étant donné le manque de recherches réalisées sur le processus de fidélisation des travailleurs du savoir, cette étude répond à un besoin de compréhension manifesté par les dirigeants du Syndicat National des Cabinets de Conseil en Management français (SYNTEC CM) quant aux raisons des cas de démission des consultants. Les résultats obtenus nous permettent de tirer des conclusions par rapport aux pratiques de mentorat dans une perspective de fidélisation des consultants. L'intention de départ volontaire de ces salariés particulièrement mobiles peut être limitée lorsque l'équipe dirigeante crée les conditions nécessaires à leur attachement affectif au cabinet. Cette étude présente clairement les pratiques de mentorat comme une option managériale aux résultats prometteurs. En effet, grâce au soutien personnel et professionnel du mentor et à la proximité issue de cette relation, le mentorat développe la valeur que les consultants ont d'eux-mêmes dans le cabinet de conseil, ce qui les lie affectivement à l'entreprise. Cette pratique prend tout son sens dès lors qu'on considère le temps que les consultants passent à l'extérieur du cabinet de conseil, en mission chez leur client. Alors que $21.6 \%$ des consultants interrogés dans cette étude ont confirmé ne pas avoir de mentor, de tels résultats laissent entrevoir le mentorat comme un levier de fidélisation pertinent pour les dirigeants des cabinets de conseil concernés.

Aussi, l'engagement envers la carrière ne semble pas participer aux effets du mentorat sur l'intention de départ. Les consultants ne souhaitent rester dans le cabinet ni pour développer leur carrière ni pour projeter un départ en vue d'assouvir un projet de carrière. Au travers de ces résultats, nous retenons le peu de relation entre le projet de carrière et l'intention de départ. Dans la mesure où la création d'une entreprise représente une autre source de motivation de départ le plus souvent communiquée par les dirigeants des cabinets, de nouvelles recherches pourraient mesurer les effets du mentorat sur ce type de projet professionnel qui justifie le départ volontaire de nombreux consultants (Tillou et Igalens, 2012). A l'issue de cette recherche, le mentorat est finalement une pratique non seulement positive à l'égard de l'attachement organisationnel mais aussi peu risquée pour les dirigeants soucieux de la fidélisation de leurs consultants.

Enfin, le rôle de l'estime de soi organisationnelle dans l'influence du mentorat rappelle l'importance à accorder aux facteurs individuels. En effet, l'efficacité des pratiques de mentorat repose aussi sur la sensibilité des consultants à la proximité du mentor et à la reconnaissance du soutien de la part des représentants de l'organisation : autant de dispositions qui peuvent varier d'un individu à l'autre. D'un point de vue managérial, ceci renvoie aux précautions nécessaires lors de la phase de sélection des consultants. Les professionnels $\mathrm{RH}$ de ces cabinets peuvent ainsi jouer un rôle majeur lors de la définition du profil des candidats à recruter. En effet, la fidélisation des consultants commence dès leur recrutement : elle repose aussi sur l'adéquation de leurs caractéristiques personnelles à leur environnement de travail.

\section{LIMITES ET PERSPECTIVES DE RECHERCHE}

Cette recherche présente des limites méthodologiques et conceptuelles. Tout d'abord, la taille de notre échantillon est limitée. Nous comptons 196 réponses apportées par des consultants représentant 15 cabinets de conseil en management français. Les recherches futures sur les effets du mentorat auprès des travailleurs du savoir devraient viser un échantillon plus large dans la mesure où l'attribution formelle et informelle du mentor dépend aussi bien des choix de pratiques de GRH de la part des équipes dirigeantes que des choix individuels des consultants. De même, il y aurait lieu de ne pas considérer les consultants comme un groupe homogène. Pour certains d'entre eux, les perspectives de carrière sont importantes alors que pour d'autres elles sont 
plus limitées, ceci pouvant affecter l'engagement de carrière (Jayasingam et Yong, 2013).

Une autre limite tient à l'adaptation du construit d'estime de soi organisationnelle aux travailleurs du savoir. Alors que les recherches justifient l'intérêt de l'analyse de l'estime de soi organisationnelle des salariés, l'échelle de mesure devrait sans doute être repensée afin d'être ajustée au contexte des travailleurs du savoir. Une autre limite est que nos données ne permettent pas de savoir si la consultation était un choix véritable de la part de nos répondants ou plutôt une décision en attendant de trouver un autre emploi. Ceci pourrait avoir influencé nos résultats.

Par ailleurs, comme de nombreuses recherches centrées sur le comportement organisationnel, nous reconnaissons des limites inhérentes au biais de méthode commune (Podsakoff, MacKenzie, Podsakoff, et Lee, 2003). En effet, parmi les différentes sources de biais existantes, notre recherche s'appuie sur deux questionnaires remplis par une même source : les consultants. Néanmoins, afin de réduire les effets de cette caractéristique du protocole, nous avons organisé une collecte de données en deux temps. Le nombre de variables mesurées au cours de cette recherche nous a contraints à associer certaines variables endogènes et exogènes lors de l'envoi du premier questionnaire. Le second questionnaire ne contenait que des items de mesure de variables endogènes. Ce type de limite renforce l'intérêt de poursuivre l'analyse explicative de l'intention de départ en développant une approche multi-niveaux. En utilisant la méthode du "Hierarchical linear modeling» (Bryk et Raudenbush, 1992), nous pourrions intégrer à l'analyse la vision des différents acteurs clés présents dans l'entourage des consultants (p.ex. l'équipe dirigeante, l'équipe de travail, le mentor, ou les clients) à l'égard du soutien perçu et des attitudes telles que l'engagement des consultants (Judge, Scott, et Ilies, 2006).

\section{Bibliographie}

Allen, D.G.; Shore, L.M.; Griffeth, R.W. (2003). "The role of perceived organizational support and supportive human resource practices in the turnover process", Journal of Management, Vol. 29, $\mathrm{N}^{\circ} 1$, p. 99-118.

Allen, T. D.; Eby, L. T.; O’Brien, K. E.; Lentz, E. (2008). “The state of mentoring research : A qualitative review of current research methods and future research implications", Journal of Vocational Behavior, Vol. 73, N³, p. 343-357.

Allen, T.D.; Eby, L.T.; Poteet, M.L.; LentZ, E.; Lima, L. (2004). "Career benefits associated with mentoring for protégés : A meta-analysis", Journal of Applied Psychology, Vol. 89, $\mathrm{N}^{\circ} 1$, p. 127-136.

Alvesson, M. (2000). "Social identity and the problem of loyalty in knowledge-intensive companies", Journal of Management Studies, Vol. 37, N8, p. 1101-1124.

Anand, N.; Gardner, H.K.; Morris, T. (2007). "Knowledgebased innovation : emergence and embedding of new practice areas in management consulting firms", Academy of Management Journal, Vol. 50, $\mathrm{N}^{\circ} 2$, p. 406-428.
Aryee, S.; Chay, Y.W. (1994). "An examination of the impact of career-oriented mentoring on work commitment attitudes and career satisfaction among professional and managerial employees", British Journal of Management, Vol. 5, N²4, p. 241-249.

Aryee, S.; TAN, K. (1992). "Antecedents and outcomes of career commitment", Journal of Vocational Behavior, Vol. 40, $\mathrm{N}^{\circ} 3$, p. 288-305.

Baranik, L. E.; Roling, E. A.; Eby, L. T. (2010). "Why does mentoring work? The role of perceived organizational support", Journal of vocational behavior, Vol. 76, $\mathrm{N}^{\circ} 3$, p. 366-373.

BATT, R. (2002). "Managing customer services : Human resource practices, quit rates, and sales growth", Academy of Management Journal, Vol. 45, N³, p. 587-597.

Bedeian, A.; Kemery, E.; Pizzolatto, A. (1991). "Career commitment and expected utility of present job as predictors of turnover intentions and turnover behaviour", Journal of Vocational Behavior, Vol. 39, p. 331-343.

Bentein, K.; Stinglhamber, F.; Vandenberghe, C. (2000). "L'engagement des salariés dans le travail», Revue québécoise de psychologie, Vol. 21, N³, p. 133-157.

Bentler, P.M.; Bonett, D.G. (1980). "Significance tests and goodness of fit in the analysis of covariance structures", Psychological Bulletin, Vol. 88, N³, p. 588-606.

Bersin \& Associates, (2009). "La gestion des talents : une aproche globale pour des besoins locaux", Research Bulletin, Vol. 4, N³8, p. 1-16.

Bishop, J.W.; Scott, K.D.; Burroughs, S.M. (2000). "Support, commitment and employee outcomes in a team environment", Journal of Management, Vol. 26, Nº6, p. 1113-1132.

Bishop, J.W.; Scott, K.D.; Goldsby, M.G.; Cropanzano, R. (2005). "A construct validity Study of commitment and perceived support variables : A multifoci approach across different team environments", Group \& Organization Management, Vol. 30, $\mathrm{N}^{\circ} 2$, p. 153-180.

BLAU, G. (1985). "The measurement and prediction of career commitment", Journal of Occupational Psychology, Vol. 58, $\mathrm{N}^{\circ} 4$, p. $277-288$.

BLAU, G. (1989). "Testing generalizability of a career commitment measure and its impact on employee turnover", Journal of Vocational Behavior, Vol. 35, Nº1, p. 88-103.

Bowling, N. A.; Eschleman, K. J.; Wang, Q.; Kirkendal, C.; Alarcon, G. (2010). Metaanalysis on the antecedents and consequences of Organization-Based Self-Esteem (OBSE). Journal Occupational and Organizational Psychology, Vol. 83, $\mathrm{N}^{\circ} 3$, p. 601-626.

Bryk, A.A.; Raudenbush, S.W. (1992). Hierarchical linear models : Applications and data analysis methods, Thousand Oaks, CA : Sage.

Carson, K. D.; Carson, P. P.; Lanford, H.; Roe, C. W. (1997). "The effects of organization-based self-esteem on workplace outcomes : An examination of emergency medical technicians”, Public Personnel Management, Vol. 26, №1, p. 139-155.

Chafetz, B.; Adair Erickson, R.; Ensell, J. (2011). "Where did your employee go ? Examining the rise in voluntary turnover during economic recoveries", Deloitte Review.

Chang, E. (1999). "Career commitment as a complex moderator of organizational commitment and turnover intention", Human Relations, Vol. 52, Nº10, p. 1257-1278. 
Chattopadhyay, P. (1999). "Beyond direct and symmetrical effects : The influence of demographic dissimilarity on organizational citizenship behaviour", Academy of Management Journal, Vol. 42, N³, p. 273-287.

Dawley, D.D.; Andrews, M.C.; Bucklew, N.S. (2008). "Mentoring, supervisor support and perceived organizational support : What matters most?", Leadership \& Organization Development Journal, Vol. 29, $\mathrm{N}^{\circ} 3$, p. 235-247.

DAY, R.; Allen, T.D. (2004). “The relationship between career motivation and self-efficacy with protégé career success", Journal of Vocational Behavior, Vol. 64, $\mathrm{N}^{\circ} 1$, p. 72-91.

Den Hartog, D.N.; BelschaK, F.D. (2007). "Personal initiative, commitment and affect at work", Journal of Occupational and Organizational Psychology, Vol. 80, º4, p. 601-622.

Eby, L. T.; Allen, T. D.; Evans, S. C.; NG, T.; DuBois, D. (2008). "Does mentoring matter? A multidisciplinary meta-analysis comparing mentored and non mentored individuals", Journal of Vocational Behavior, Vol. 72, º2, p. 254-267.

Ellemers, N.; de Gilder, D.; van den Heuvel, H. (1998). "Career-oriented versus team-oriented commitment and behavior at work", Journal of Applied Psychology, Vol. 83, N 5 , p. 717-730.

FAgenson, E.A. (1989). “The mentor advantage : Perceived career/ job experiences of protégés versus non protégés”, Journal of Organizational Behavior, Vol. 10, N4, p. 309-320.

Ferris, D.L.; Brown, D.J.; Heller, D. (2009). “Organizational supports and organizational deviance: The mediating role of organization-based self-esteem", Organizational Behavior and Human Decision Processes, Vol. 108, N² 2 , p. 279-286.

Fleig-Palmer, M.M. (2009). The impact of mentoring on retention through knowledge transfer, affective commitment, and trust, Unpublished doctoral dissertation, University of Nebraska.

Gardner, D.G.; Pierce, J.L. (1998). "Self-esteem and self-efficacy within the organizational context : An empirical examination", Group \& Organization Management, Vol. 23, №1, p. $48-70$.

Ghosh, R.; Reio JR, T. G. (2013). "Career Benefits Associated with Mentoring for Mentors : A Meta-Analysis”, Journal of Vocational Behavior, Vol. 83, $\mathrm{N}^{\circ} 1$, p. 106-116.

Haggard, D. L.; Dougherty, T. W.; Turban, D. B.; Wilbanks, J. E. (2011) "Who is a mentor? Alternative definitions and implications for research", Journal of Management. Vol. 37, p. 280-304.

HALl, D. (1971). "A theoretical model of career subidentity development in organizational settings", Organizational Behavior and Human Performance, Vol. 6, p. 50-76.

Hartmann, N. N.; Rutherford, B. N.; Hamwi, G. A.; Friend, S.B. (2013). "The effects of mentoring on salesperson commitment”, Journal of Business Research, Vol. 66, $N^{\circ} 11$, p. 2294-2300.

Horwitz, F.M.; Heng, C.T.; Quazi H.A. (2003). "Finders, keepers? Attracting, motivating and retaining knowledge workers", Human Resource Management Journal, Vol. 13, N 4 , p. 23-44.

Hunt, D. M.; Michael, C. (1983). "Mentorship : A career training and development tool", Academy of Management Review, Vol.8, N³, p. 475-485.
JAros, S. (1997). “An assessment of Meyer and Allen's (1991) three-commitment model of organizational commitment and turnover intentions", Journal of Vocational Behavior, Vol. 51, N³, p. 319-337.

Jayasingam, S.; Yong, J. R. (2013). "Affective commitment among knowledge workers : the role of pay satisfaction and organization career management", International Journal of Human Resource Management, Vol. 24, º20, p. 3903-3920.

Joiner, T. A.; Bartram, T.; Garreffa, T. (2004). “The effects of mentoring on perceived career success, commitment and turnover intentions", Journal of American Academy of Business, Vol. 5, p. 164-153.

Judge, T.A.; Scott, B.A.; Ilies, R. (2006). "Hostility, job attitudes, and workplace deviance : Test of a multilevel model", Journal of Applied Psychology, Vol. 91, N¹, p. 126-138.

Kenoe, R. R.; Wright, P. M. (2013). "The impact of high performance human resource practices on employees' attitudes and behaviors", Journal of Management, Vol. 39, N², p. 366-391.

Kinnear, L.; Sutherland, M. (2000). "Determinants of organizational commitment amongst knowledge workers", South African Journal of Business Management, Vol. 32, $\mathrm{N}^{\circ} 2$, p. 106-111.

Koberg, C. S.; Boss, R.W.; Goodman, E. (1998). "Factors and outcomes associated with mentoring among health-care professionals", Journal of Vocational Behavior, Vol. 53, p. 58-72.

KRAM, K.E. (1983). "Phases of the mentor relationship", Academy of Management Journal, Vol. 26, $\mathrm{N}^{\circ} 4$, p. 608-625.

KRAM, K.E. (1985). Mentoring at work: Developmental relationships in organizational life, Glenview, IL : Scott Foresman.

KRAM, K.E. (1986). "Mentoring in the workplace”, Dans D. T. Hall (Ed.), Career development in organizations, p. 160-201, San Francisco : Jossey-Bass, 268 p.

LeE, J.; PecceI, R. (2007). "Perceived organizational support and affective commitment : The mediating role of organizationbased self-esteem in the context of job insecurity", Journal of Organizational Behavior, Vol. 28, $\mathrm{N}^{\circ} 6$, p. 661-685.

Little, T.D.; Cunningham, W.A.; Shahar, G.; Widaman, K.F. (2002). "To parcel or not to parcel : Exploring the question, weighing the merits", Structural Equation Modeling, Vol. 9, p. 152-173.

MacKinnon, D.P.; Lockwood, C.M.; Williams, J. (2004). "Confidence limits for the indirect effect: Distribution of the product and resampling methods", Multivariate Behavioral Research, Vol. 39, $\mathrm{N}^{\circ} 1$, p. 99-128.

Meyer, J.P.; Allen, N.J. (1997). Commitment in the workplace: Theory, research and application, Newbury Park, CA : Sage.

Meyer, J.P.; Allen, N.J.; Smith, C.A. (1993). “Commitment to organizations and occupations : Extension and test of a threecomponent conceptualization", Journal of Applied Psychology, Vol. 78, N4, p. 538-551.

Meyer, J. P.; Becker, T. E.; Vandenberghe, C. (2004). "Employee commitment and motivation : A conceptual analysis and integrative model", Journal of Applied Psychology, Vol. 89, N6, p. 991-1007.

Meyer, J.P.; Stanley, D.J.; Herscovitch, L.; Topolnytsky, L. (2002). "Affective, continuance and normative commitment to the organization : A meta-analysis of antecedents, correlates and consequences", Journal of Vocational Behavior, Vol. 61, Nº1, p. 20-52. 
Payne, S.C.; Huffman, A.H. (2005). "A longitudinal examination of the influence of mentoring on organizational commitment and turnover", Academy of Management Journal, Vol. 48, $\mathrm{N}^{\circ} 1$, p. 158-168.

Pierce, J.L.; Gardner, D.G. (2004). "Self-esteem within the work and organizational context : A review of the organization-based self-esteem literature", Journal of Management, Vol. 30, N5, p. 591-622.

Pierce, J.L.; Gardner, D.G.; Cummings, L.L.; Dunham, R.B. (1989). "Organization-based self-esteem : construct definition, measurement, and validation", Academy of Management Journal, Vol. 32, $\mathrm{N}^{\circ} 3$, p. 622-648.

Pierce, J.L.; Gardner, D.G.; Dunham, R.B.; Cummings, L.L. (1993). "Moderation by organization-based self-esteem of role condition-employee response relationships", Academy of Management Journal, Vol. 36, $\mathrm{N}^{\circ} 2$, p. 271-288.

Podsakoff, P.M.; MacKenzie, S.B.; Podsakoff, N.P.; Lee, J.Y. (2003). "Common method biases in behavioral research : A critical review of the literature and recommended remedies", Journal of Applied Psychology, Vol. 88, N5, p. 879-903.

Ragins, B.R.; Cotton, J.L. (1999). "Mentor functions and outcomes: A comparison of men and women in formal and informal mentoring relationships", Journal of Applied Psychology, Vol. 84, N4, p. 529-550.

Ragins, B.R.; Cotton, J.L.; Miller, J.S. (2000). "Marginal mentoring: The effects of type of mentor, quality of relationship, and program design on work and career attitudes", Academy of Management Journal, Vol. 43, N6, p. 1177-1194.

Ragins, B.R.; McFarlin, D.B. (1990). "Perceptions of mentor roles in cross-gender mentoring relationships", Journal of Vocational Behavior, Vol. 37, $\mathrm{N}^{\circ} 3$, p. 321-339.

Rhoades, L.; Eisenberger, R. (2002). "Perceived organizational support : A review of the literature", Journal of Applied Psychology, Vol. 87, N4, p. 698 -714.

Richard, O.C.; IsMAIL, K.M.; BHUIAN, S.N.; TAYLOR, E.C. (2009). "Mentoring in supervisor-subordinate dyads : Antecedents, consequences, and test of a mediation model of mentorship", Journal of Business Research, Vol. 62, Nº11, p. 1110-1118.

Romzeк, B.S. (1989). "Personal consequences of employee commitment”, Academy of Management Journal, Vol. 32, N³, p. 649-661.

SARVARY, M. (1999). "Knowledge management and competition in the consulting industry", California Management Review, Vol. 41, N², p. 95-107.

SCANDURA, T.A. (1992). "Mentorship and career mobility : An empirical investigation", Journal of Organizational Behavior, Vol.13, N², p. 169-174.

Sun, L.; Aryee, S.; LaW, K. S. (2007). "High-performance human resource practices, citizenship behavior, and organizational performance : A relational perspective", Academy of Management Journal, Vol. 50, N³, p. 558-577.

Swart, J.; KInNIE, N. (2003). "Sharing knowledge in knowledgeintensive firms", Human Resource Management Journal, Vol. 13, N², p. 60-75.

Tett, R. P.; Meyer, J. P. (1993). “Job satisfaction, organizational commitment, turnover intention, and turnover : Path analyses based on meta-analytic findings", Personnel Psychology, Vol. 46, $\mathrm{N}^{\circ}$ 2, p. 259-293.
Tillou, C.; Igalens, J. (2012). «Pourquoi les consultants français quittent-ils leurs employeurs?", Revue de Gestion des Ressources Humaines, $\mathrm{N}^{\circ} 84$, p. 22-43.

UDEH, I.E.; OMAR, A. (2009). "Strategic versus gratuitous mentoring : A preliminary investigation", Proceedings of the Academy of Strategic Management, Vol. 8, p. 44-47.

Vandenberghe, C.; Bentein, K.; Stinglhamber, F. (2004). "Affective commitment to the organization, supervisor, and work group : Antecedents and outcomes", Journal of Vocational Behavior, Vol. 64, $\mathrm{N}^{\circ} 1$, p. 47-71.

Viator, R.E.; SCANDURA, T.A. (1991). "A study of mentor-protégé relationships in large public accouting firms", Accounting Horizons, Vol. 5, p. 20-30.

Wanberg, C.R.; Welsh, E.; Kammeyer-Mueller, J.D. (2007). "Protégé and mentor self-disclosure : Levels and outcomes within formal mentoring dyads in a corporate context", Journal of Vocational Behavior, Vol. 70, p. 398-412.

Werr, A.; Stjernberg, T. (2003). "Exploring management consulting firms as knowledge systems", Organization Studies, Vol. 24, p. 881-908. 